\title{
Wide-field phase imaging for the endoscopic detection of dysplasia and early-stage esophageal cancer
}

C. R. M. Fitzpatrick, G. S. D. Gordon, T. W. Sawyer, T. D. Wilkinson, S. E. Bohndiek

C. R. M. Fitzpatrick, G. S. D. Gordon, T. W. Sawyer, T. D. Wilkinson, S. E. Bohndiek, "Wide-field phase imaging for the endoscopic detection of dysplasia and early-stage esophageal cancer ,"Proc. SPIE 10470, Endoscopic Microscopy XIII, 1047015 (28 February 2018); doi: 10.1117/12.2290910

SPIE. Event: SPIE BiOS, 2018, San Francisco, California, United States 


\title{
Wide-field phase imaging for the endoscopic detection of dysplasia and early-stage esophageal cancer
}

\author{
C. R. M. Fitzpatrick ${ }^{\mathrm{a}}$, G. S. D. Gordon ${ }^{\mathrm{a}}$, T. W. Sawyer ${ }^{\mathrm{b}, \mathrm{c}}$, T. D. Wilkinson ${ }^{\mathrm{a}}$, and S. E. \\ Bohndiek $^{\mathrm{b}}$ \\ ${ }^{a}$ Department of Electrical Engineering, University of Cambridge, JJ Thomson Avenue, Cambridge CB3 0FA, UK \\ ${ }^{\mathrm{b}}$ Department of Physics and CRUK Cambridge Institute, University of Cambridge, Cambridge, UK \\ ${ }^{\mathrm{c}}$ College of Optical Sciences, The University of Arizona, Tucson, Arizona 85721, USA
}

\begin{abstract}
Esophageal cancer has a 5-year survival rate below 20\%, but can be curatively resected if it is detected early. At present, poor contrast for early lesions in white light imaging leads to a high miss rate in standard-ofcare endoscopic surveillance. Early lesions in the esophagus, referred to as dysplasia, are characterized by an abundance of abnormal cells with enlarged nuclei. This tissue has a different refractive index profile to healthy tissue, which results in different light scattering properties and provides a source of endogenous contrast that can be exploited for advanced endoscopic imaging. For example, point measurements of such contrast can be made with scattering spectroscopy, while optical coherence tomography generates volumetric data. However, both require specialist interpretation for diagnostic decision making. We propose combining wide-field phase imaging with existing white light endoscopy in order to provide enhanced contrast for dysplasia and early-stage cancer in an image format that is familiar to endoscopists. Wide-field phase imaging in endoscopy can be achieved using coherent illumination combined with phase retrieval algorithms. Here, we present the design and simulation of a benchtop phase imaging system that is compatible with capsule endoscopy. We have undertaken preliminary optical modelling of the phase imaging setup, including aberration correction simulations and an investigation into distinguishing between different tissue phantom scattering coefficients. As our approach is based on phase retrieval rather than interferometry, it is feasible to realize a device with low-cost components for future clinical implementation.
\end{abstract}

Keywords: cancer, capsule endoscopy, dysplasia, early detection, phase retrieval

\section{INTRODUCTION}

The 5-year survival rate for esophageal cancer rises from under $20 \% 1,2$ to around $80 \%$ when it is detected early. ${ }^{3}$ Patients with Barrett's Esophagus, an inflammatory condition associated with acid reflux, have an elevated risk of developing esophageal cancer and so undergo endoscopic surveillance with the goal of detecting neoplasia, a term which encompasses early-stage cancer and its precursor, dysplasia. However, the current standard of care has a relatively low sensitivity for neoplasia of 40-64\%. ${ }^{4}$ It comprises white light imaging paired with taking four systematic biopsies every $2 \mathrm{~cm}$ throughout the Barrett's segment to mitigate the risk of missing early lesions during endoscopic inspection, making the process costly and prone to sampling error.

Advanced imaging techniques could improve the standard of care by increasing diagnostic sensitivity. Research in this area has been discussed in several recent reviews ${ }^{4-7}$ and thresholds for endoscopic imaging performance have been proposed above which systematic biopsies would no longer be required. ${ }^{6}$ While techniques including narrow band imaging and confocal laser endomicroscopy meet these thresholds, ${ }^{6}$ image interpretation requires specialist training and clinical use of these techniques is not widespread.

Some advanced imaging techniques derive label-free contrast from the morphological changes that occur during neoplastic transformation. Label-free contrast is desirable because it eliminates the risks, procedural steps and regulatory hurdles associated with the development of new contrast agents. Instead, contrast can be obtained based on the increased abundance of enlarged cell nuclei in diseased tissue, which cause stronger light scattering than healthy tissue. ${ }^{8}$ This provides contrast in optical coherence tomography (OCT), ${ }^{9}$ and can be probed more directly using scattering spectroscopy ${ }^{10}$ or angle-resolved low-coherence tomography ${ }^{11}$ to quantify changes in nuclear size and density. Tethered capsule endoscopes with OCT-based imaging can produce high-resolution, 
depth-resolved maps of tissue structure, but face the challenges of automating what is currently a time-consuming image interpretation procedure that requires specialist training. ${ }^{12}$ Conversely, while endoscopic polarized scattering spectroscopy (EPSS) lacks depth resolution, it has been demonstrated to distinguish between healthy and dysplastic tissue with a simple thresholding approach. ${ }^{13}$ All scattering-based approaches to date require scanning mechanisms to assess large areas of tissue, which adds cost and complexity to data acquisition.

Here, we explored the concept of applying holographic techniques to the endoscopic investigation of light scattering from tissue with a view to implementation in a low-cost capsule form. The output is wide-field phase maps that could be co-registered with the familiar view obtained using endoscopic white light imaging. Wide-field quantitative phase imaging (QPI) has been applied to the assessment of malignancy in ex-vivo tissue, ${ }^{14}$ but current interferometric QPI techniques are not compatible with endoscopy. Our proposed approach does not require any moving parts, and is well-suited to assessing the entire endoscopic field of view because phase information can be used to correct aberrations and perform lensless working distance adjustments. ${ }^{15}$ Well-characterized, thresholded phase scattering data overlaid on familiar white-light imaging could provide intuitive contrast for neoplastic transformation with minimal clinical training requirements.

The goal of this work was to develop a simulation framework to evaluate key aspects of producing a wide-field, low-cost phase imaging capsule, and to link simulation results with experimental measurements. The presented results are based on use of a wide-field phase imaging system that is compatible with the size and cost constraints of a tethered capsule endoscope. Agreement between simulation and experimental work, demonstrations of aberration correction and a first look at distinguishing between different levels of tissue scattering, all provide evidence for the future potential of this approach.

\section{METHODS}

\subsection{4 optical system}

A wide-field phase measurement system for use within a capsule endoscope needs to be more robust than interferometric approaches and also suitable for miniaturised implementation. With this in mind, the first system that was simulated was a $4 \mathrm{f}$ system, so named because it spans four lens focal lengths (f). It is shown schematically in Figure 1. Propagation from the object plane to the image plane can be completely described by two 2D Fourier transforms, making it an intuitive starting point for wide-field phase retrieval. The system has a magnification of -1 (i.e. image inversion) when the two lenses have equal focal lengths; this is true for all presented work. Changing the ratio of focal lengths adjusts the magnification, which can be done to accommodate fields of view larger than the sensor area. Figure 1 indicates how a $4 \mathrm{f}$ phase retrieval system might fit inside a capsule, where the anticipated length and diameter are $25 \mathrm{~mm}$ and $11 \mathrm{~mm}$ respectively. While initial experiments were carried out using $\mathrm{f}=100 \mathrm{~mm}$, lenses with $3 \mathrm{~mm}$ focal lengths and sensors with $1 \times 1 \mathrm{~mm}$ footprints are commercially available, indicating that miniaturization is feasible.

Sensor 1

(Fourier plane)

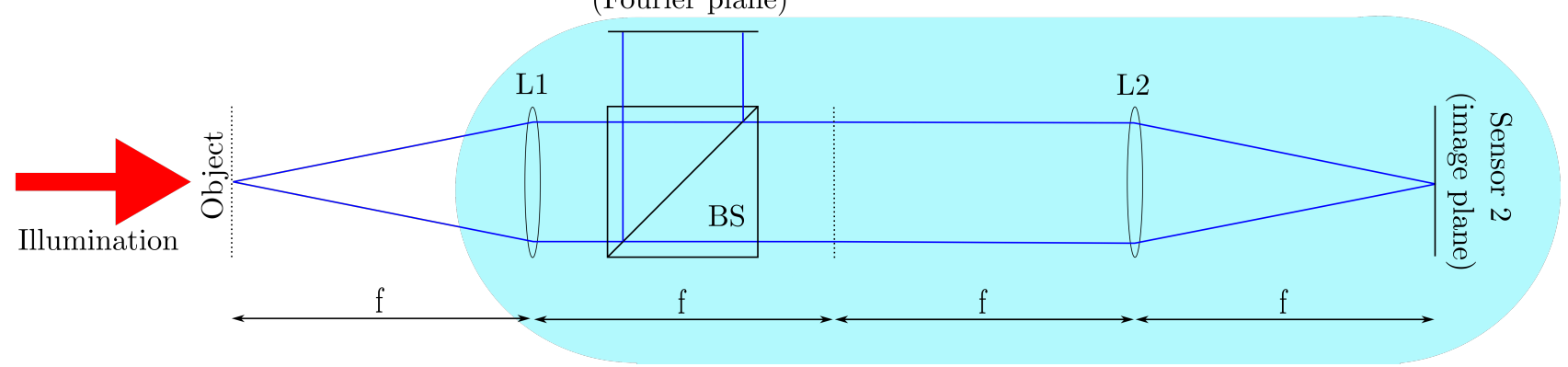

Figure 1. Schematic diagram of $4 \mathrm{f}$ optical system. Coherent light from the back-illuminated object plane is divided between Fourier plane and image sensors. The blue shape indicates how components could be housed inside a capsule endoscope.

Figure 2 shows a photograph of the $4 \mathrm{f}$ system that was used for initial experiments. $\mathrm{f}=100 \mathrm{~mm}$ lenses (Thorlabs AC254-100-A-ML) were chosen to aid alignment and provide ample inter-component access, and a $100 \mu \mathrm{m}$ 
pinhole (Thorlabs $\mathrm{P} 100 \mathrm{H}$ ) was positioned at the object plane. It was back-illuminated by a collimated $633 \mathrm{~nm}$ HeNe laser beam (SpectraPhysics 155SL) with $1 \mathrm{~mm}$ diameter, delivered with two steering mirrors. Ultimately, the $\mathrm{HeNe}$ will be replaced with a low-cost laser diode that could fit inside a capsule. A wavelength of $633 \mathrm{~nm}$ was determined to be suitable for initial experiments because its tissue penetration depth of at least $0.5 \mathrm{~mm}^{16}$ is compatible with the average thickness of the esophageal epithelium, ${ }^{17}$ where neoplasia typically occurs. The light was divided between the two sensors using a non-polarizing 50:50 beam splitter (Thorlabs CM1-BS013). The sensors were mounted on 3-axis translatable cage plates (Thorlabs CXYZ05/M), enabling fine-tuning of their alignment based on live sensor feedback.

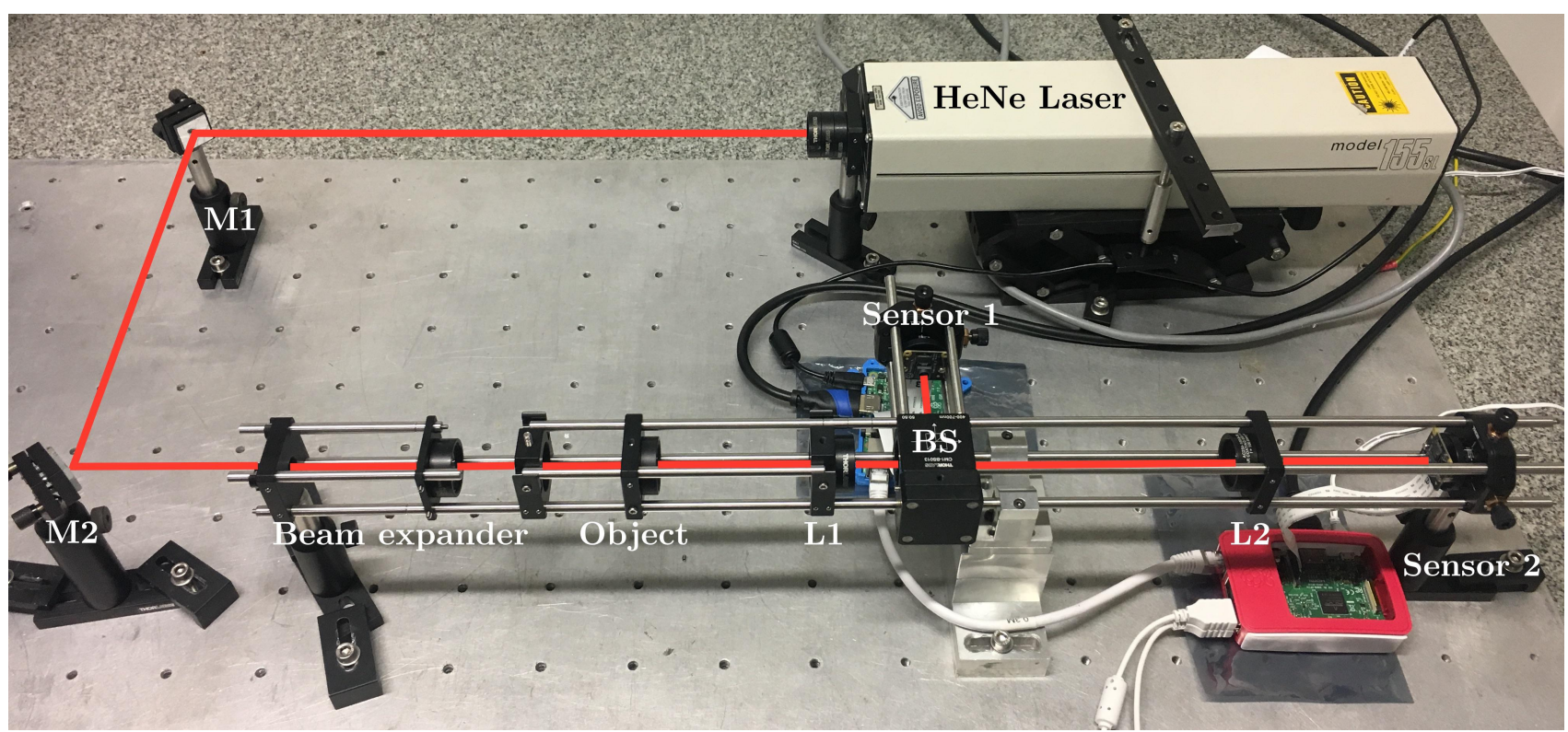

Figure 2. Labelled photograph of 4 f optical system. The delivery of laser illumination using two steering mirrors is shown in addition to the components in Figure 1. The focal length of L1 and L2 is $100 \mathrm{~mm}$.

Intensities at the $2 \mathrm{f}$ and $4 \mathrm{f}$ planes were recorded using Raspberry Pi v2.0 NoIR camera modules with their lenses removed. These modules contain Sony IMX219 8-megapixel sensors with $1.12 \mu \mathrm{m}$ pixels. Data acquisition with both sensors was controlled from a single Raspberry Pi computer using an http server arrangement, and Python's pycamera module was used to control acquisition parameters. The raw 10-bit values associated with the red component of the sensor's Bayer filter were extracted and demosaiced in order to access the maximum available dynamic range and avoid the nonlinearities introduced by on-board gamma correction.

\subsection{Propagation simulation}

Beam propagation simulations were developed to model the expected intensity and phase distributions at the sensor planes for a given complex field at the object plane. Propagation simulation also forms part of the phase retrieval approach described in Section 2.3. The simulations apply Fourier optics concepts ${ }^{18}$ in MATLAB using 2D discrete Fourier transforms and associated in-built functions.

The $4 \mathrm{f}$ system was first modelled using Fraunhofer propagation. The complex field associated with the object plane was initialised to match the pixel array size of the sensors, then successive discrete 2D Fourier transforms were calculated to propagate to the $2 \mathrm{f}$ and $4 \mathrm{f}$ planes. The crucial aspect of this calculation is determining the correct scaling between the $2 \mathrm{f}$ and $4 \mathrm{f}$ planes in order to correctly interpret the intensities measured on the sensors. This is because both sensors have identically-sized pixels, while the 2D Fourier transform of a field typically has array elements of a different size to those of the original field. To determine the expected intensity distribution at the $2 \mathrm{f}$ sensor, the field must be cropped or padded to match the size of the sensor. This can be done using the following expressions for the scaled side length $L_{2 f}$ and element size $\Delta L_{2 f}{ }^{19}$ in the Fourier domain: 


$$
L_{2 f}=\frac{\lambda f}{\Delta L_{4 f}} \quad \text { and } \quad \Delta L_{2 f}=\frac{\lambda f}{L_{4 f}} .
$$

The optical components must be in a $4 \mathrm{f}$ configuration in order for Fraunhofer propagation to be valid; using the Fresnel approximation extends the validity of the propagation simulations to permit the movement of components away from their $4 \mathrm{f}$ positions. ${ }^{18}$ This provides more flexibility to optimise the optical design within a spaceconstrained system. Once the Fraunhofer propagation results were verified by comparison to the analytical test case of a pinhole at the target position, the simulations were then extended to incorporate Fresnel propagation.

\subsection{Phase retrieval}

The goal of phase retrieval is to reconstruct a wide-field phase image from a set of measured intensities. Making multiple intensity measurements samples the light at different points in its propagation, allowing the additional phase information to be determined. The Gerchberg-Saxton algorithm ${ }^{20}$ is an iterative phase retrieval approach that is known to converge for the case of two $2 \mathrm{D}$ intensity arrays with a $2 \mathrm{f}-4 \mathrm{f}$ relationship. ${ }^{21}$ The algorithm implemented in MATLAB is shown schematically in Figure 3; it involves iteratively propagating between the two sensor planes, each time retaining the estimated phase and inserting the measured amplitude. Once the algorithm has converged, the phase component of the complex field at the image plane can be taken to represent the phase array at the object plane, thus providing phase information about the imaging target.

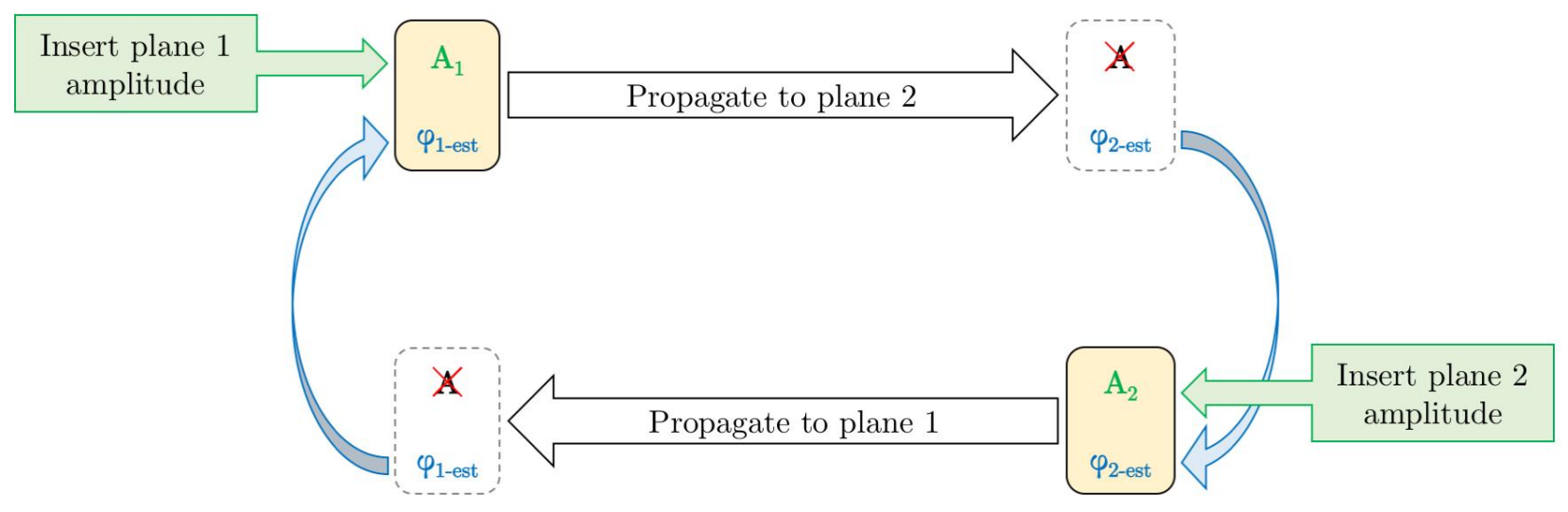

Figure 3. Schematic diagram of the Gerchberg-Saxton algorithm. Plane 1 and 2 are the $2 \mathrm{f}$ and $4 \mathrm{f}$ planes for the majority of the presented work, but can be planes related by a wide variety of linear transforms. ${ }^{22}$

This algorithm has been implemented in MATLAB and is successfully applied in Sections 3.1 and 3.2 to inputs based on simulations and on experimental measurements. As described in Section 2.2, it is crucial to determine the correct scaling between the two planes in order for the phase retrieval algorithm to successfully converge.

\subsection{Aberration correction}

Optical aberrations are commonly characterized by lens designers in terms of 'wavefront error'. In essence, this is the spatially-varying phase deviation from a perfect spherical wavefront. As our approach works with complex fields instead of just intensities, it is possible to correct for aberrations by incorporating the inverse phase transformation to the field in the spatial frequency domain. ${ }^{18}$ This is especially valuable for aberrations that cannot be corrected with intensity information alone, such as spherical aberration. In future work, this approach will be incorporated into the propagation steps of the Gerchberg-Saxton algorithm (see Figure 3) in order to correct for aberrations in the second lens of a wide-field phase imaging system such as the one presented. 


\subsection{Scattering data generation}

In addition to well-defined input fields such as pinholes or other shaped apertures, data sets were generated to be more representative of the phase scattering that would be observed from tissue. Appropriate scattering distributions were determined from experimental measurements of a tissue mimicking phantom with known phase scattering made using a fiber-based phase imaging system. A Gaussian copula was fit to experimental data in order to simulate complex fields that matched the dimensions of the sensor while preserving the distribution and dependence between the intensity and phase components of the input data. Based on the copula fit results, data sets with representative random phase and intensity components were generated for two different scattering coefficients: $\mu_{s}=0.5 \mathrm{~cm}^{-1}$ and $2.0 \mathrm{~cm}^{-1}$.

\section{RESULTS}

\subsection{Wide-field phase: Simulation and experiment}

The simplest phase imaging target that was tested was a $100 \mu \mathrm{m}$ pinhole was selected as the first phase imaging target. This enabled the propagation simulations to be checked against the analytical result, as the Fourier transform of a $2 \mathrm{D}$ circle function is known to be a sombrero-like jinc function. ${ }^{19}$ With the propagation code verified, the simulated intensity distributions were used as inputs to the phase retrieval code. The retrieved phase was confirmed as matching that from the propagation simulations. Additionally, comparable phase retrieval results were achieved using Fresnel propagation in simulations where the $2 \mathrm{f}$ sensor was positioned $10 \%$ closer to the first lens.

Next, experimental measurements were made of the phase distribution at the $2 \mathrm{f}$ plane with the pinhole backilluminated. Figure 4(a) shows the optical arrangement used to acquire experimental measurements of intensity (Fig 4(b)), which were then fed into the Gerchberg-Saxton algorithm to retrieve the phase information. Figure $4(\mathrm{c})$ shows good agreement between the simulated and experimentally meausred phase distributions.

a) 4f schematic

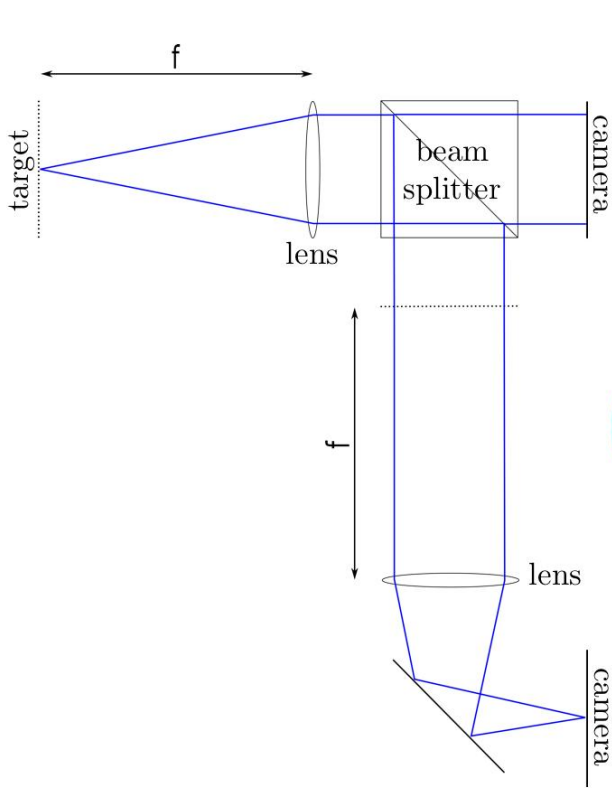

b) Measured intensities

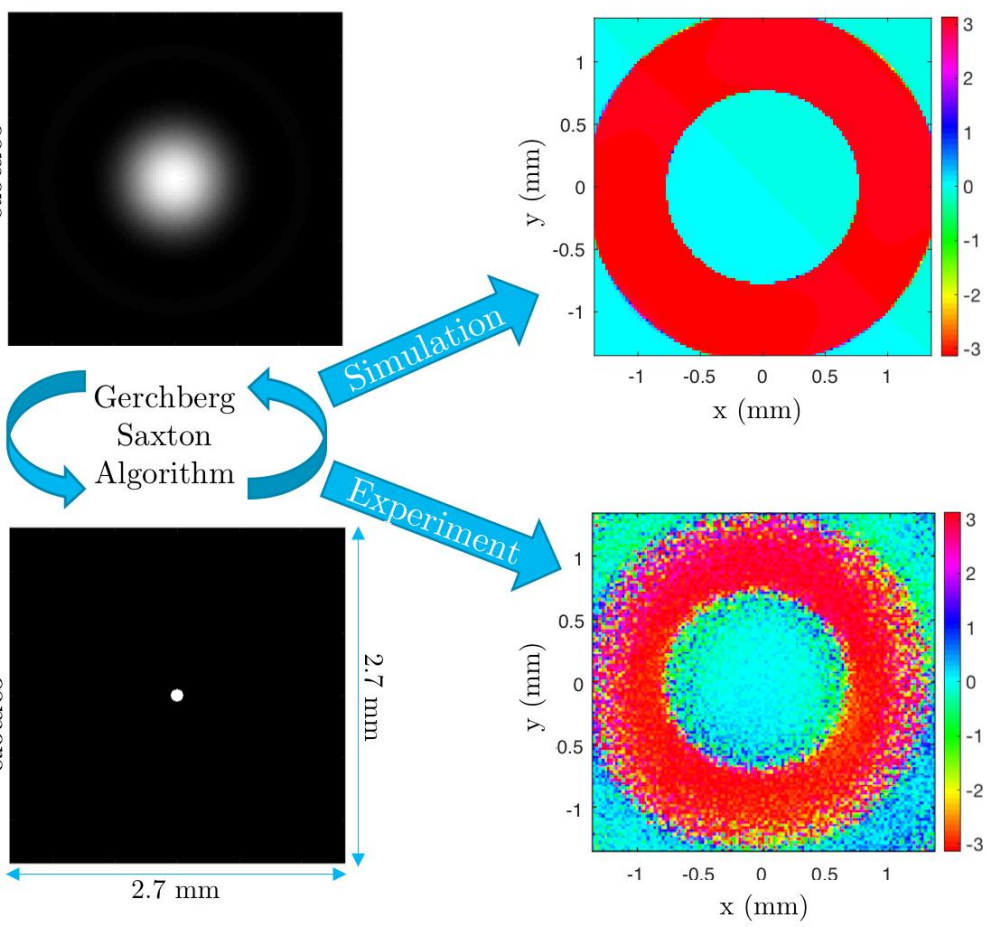

Figure 4. (a) Schematic diagram of the $4 \mathrm{f}$ system, indicating the sensor positions used to acquire the experimentallymeasured images shown in (b). (c) The wide-field phase associated with the $2 \mathrm{f}$ plane, as recovered from simulated (upper) and experimentally-measured (lower) intensity distributions. 


\subsection{Scattering simulation}

Complex fields representing scattering tissue phantoms were generated as described in Section 2.5. These fields were used as inputs to the $4 \mathrm{f}$ system propagation simulation in order to determine the associated $2 \mathrm{f}$ and $4 \mathrm{f}$ intensity distributions. The phase distributions in Figure 5 show that phase retrieval on these simulated intensities matched well with the phase distributions determined from the propagation, and that the two cases of can be distinguished from each other. The 2D phase arrays in Figure 5 show a subset of the $2 \mathrm{D}$ phase array obtained in each case.

a) Low scattering $\left(\mu_{\mathrm{s}}=0.5 \mathrm{~cm}^{-1}\right)$
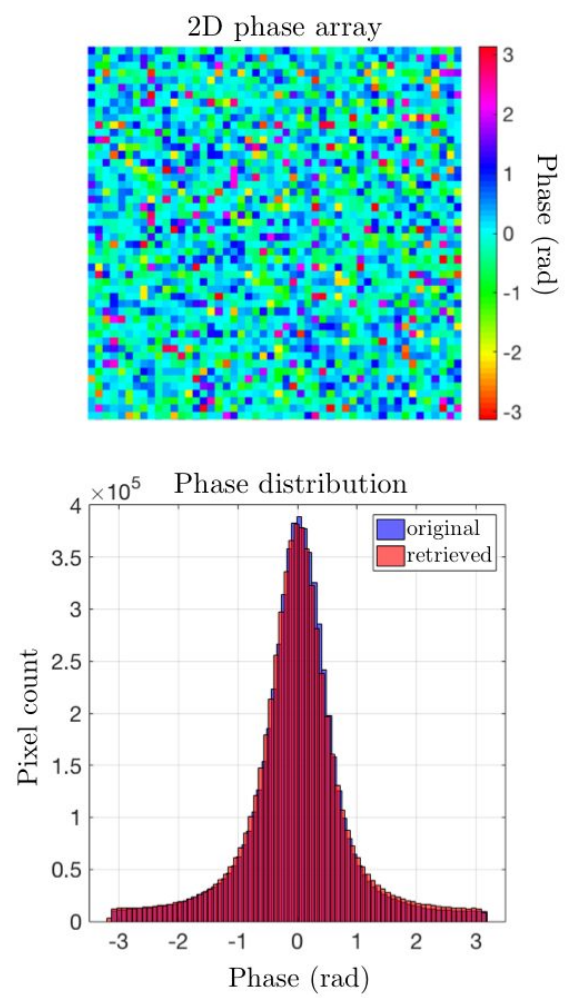

b) High scattering $\left(\mu_{\mathrm{s}}=2.0 \mathrm{~cm}^{-1}\right)$
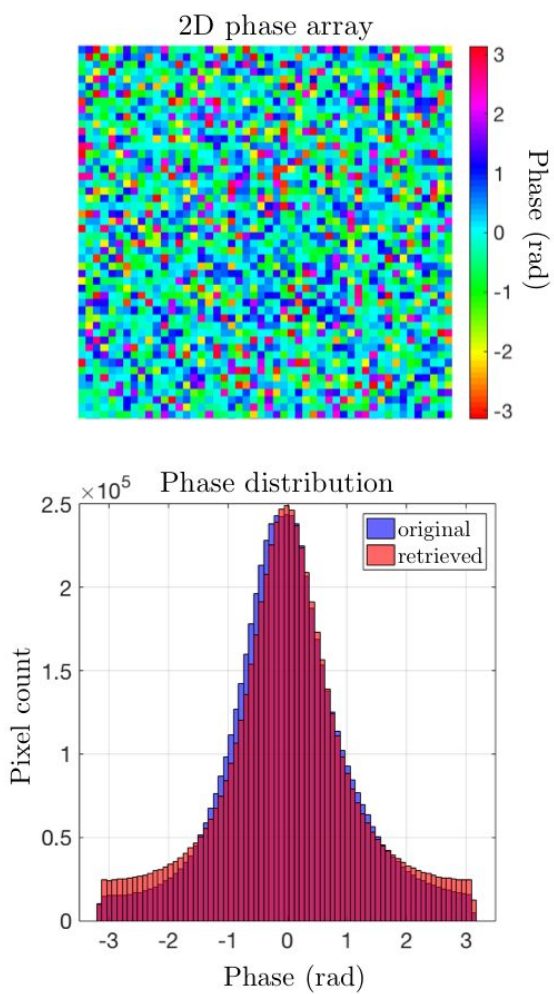

Figure 5. Retrieved phase arrays and distributions for input fields based on tissue phantoms with (a) low scattering and (b) high scattering. The displayed arrays are a subset of the total field, while the distributions are histograms of the entire data sets.

\subsection{Aberration correction}

Aberration correction simulations were conducted using an image of a number 5 with a flat associated phase. With no aberrations in the simulation, the intensity distribution at the image plane was as shown in Figure 6(a). During the next propagation simulation, a primary spherical aberration was applied at the first lens. This was implemented by multiplying the field in spatial frequency domain with a Zernike $\mathrm{Z}_{11}$ phase distribution that was scaled to a maximum phase deviation of $500 \pi$. The resulting aberrated image plane is shown in Figure $6(\mathrm{~b})$. To correct the aberration, the complex field at the $4 \mathrm{f}$ plane was propagated backwards to the spatial frequency domain, where the inverse phase transformation was applied to the field, then propagated forwards again to the image plane. The result is shown in Figure 6(c). 
a) Ideal image

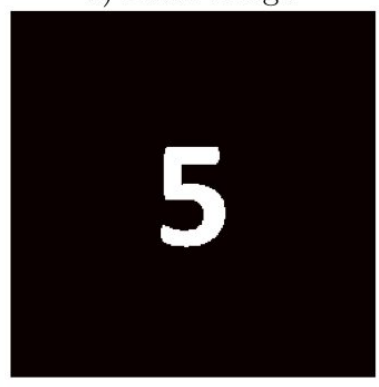

b) Aberrated image

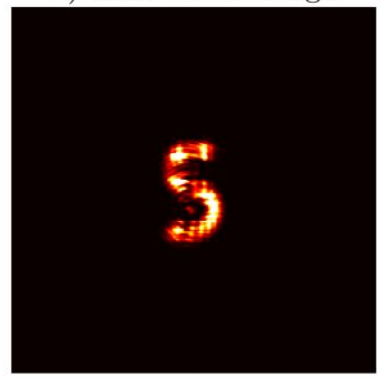

c) Corrected image

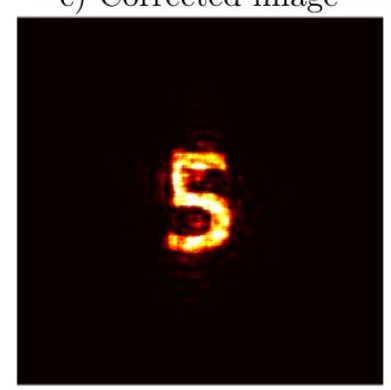

Figure 6. Intensities at the $4 \mathrm{f}$ plane with (a) no aberration in the propagation simulation, (b) $500 \pi$ magnitude primary spherical aberration and (c) after aberration correction was performed on image (b).

\section{DISCUSSION}

The goal of this work was to develop a simulation framework to evaluate key aspects of producing a wide-field, low-cost phase imaging capsule, and to link simulation results with experimental measurements. Agreement between simulated and experimentally-determined phase profiles was demonstrated over a $2.7 \mathrm{~mm} \times 2.7 \mathrm{~mm}$ square area, based on a measurement of a $100 \mu \mathrm{m}$ pinhole target. Phase retrieval was also successfully simulated for input fields representing scattering tissue phantoms.

While promising, there are key limitations to these initial tests. Firstly, the test system had a focal length of $100 \mathrm{~mm}$, which is considerably larger than a feasible endoscopic device. However, it can be determined using the expressions in equation 1 that to match the scaling of the $2 \mathrm{f}$ and $4 \mathrm{f}$ planes for the current sensors, the focal length would need to be $6 \mathrm{~mm}$. Ultimately, a ratio of focal lengths will be determined that fits within the capsule and provides the required demagnification to maximise the field of view. The exact positioning of the components is flexible due to our ability to incorporate Fresnel propagation in the data processing pipeline. A second key limitation is that an endoscopic system must be able to evaluate light reflected from tissue rather than operating in transmission like the current system. An upgraded test system that incorporates reflection-mode measurement would facilitate ex-vivo testing of thick tissue samples and tissue phantoms.

To move towards an experimental prototype of a wide-field endoscopic phase imaging system, specialized optical and mechanical components will be required, along with further developments in data acquisition and phase retrieval algorithms. Our established simulation framework now provides a basis from which to explore the parameter space of the design.

\section{CONCLUSIONS}

An endoscopic implementation of wide-field phase imaging could be used to provide clinicians with an intuitive view of the surface scattering properties of tissue. This would have diagnostic value because scattering is strongly linked to the development of dysplasia and early-stage esophageal cancer. We are developing a simulation framework and experimental platform to evaluate key implementation considerations of the proposed wide-field capsule approach, including aberration correction, tolerance to sensor position movement and sensitivity to biologically relevant scattering coefficients. Initial results show good agreement between theory and experiment; next steps will include progressing the design to include reflection mode measurements, investigating miniaturisation and performing extensive measurements on tissue samples and phantoms.

\section{ACKNOWLEDGMENTS}

This work was primarily funded by a pump-priming award from the Cancer Research UK Cambridge Cancer Centre Early Detection Programme (A20976). The authors would also like to acknowledge further CRUK funding (C14303/A17197, C47594/A16267, C47594/A21102) and the European Union's Seventh Framework Programme under grant number FP7-PEOPLE-213-CIG-630729. The authors would like to thank Dr. James Joseph for productive discussions regarding scattering tissue phantoms. 


\section{REFERENCES}

[1] Cancer Research UK, "Oesophageal cancer survival statistics." http://www.cancerresearchuk.org/healthprofessional/cancer-statistics/statistics-by-cancer-type/oesophageal-cancer/survival (Jan. 2018).

[2] National Cancer Institute, "SEER Cancer Stat Facts: Esophageal Cancer." https://seer.cancer.gov/statfacts/html/esoph.html (Jan. 2018).

[3] Barbour, A. P., Jones, M., Brown, I., Gotley, D. C., Martin, I., Thomas, J., Clouston, A., and Mark Smithers, B., "Risk Stratification for Early Esophageal Adenocarcinoma: Analysis of Lymphatic Spread and Prognostic Factors," Annals of Surgical Oncology 17(9), 2494-2502 (2010).

[4] Sturm, M. B. and Wang, T. D., "Emerging optical methods for surveillance of Barretts oesophagus," Gut 64(11), 1816-1823 (2015).

[5] Robles, L. Y., Singh, S., and Fisichella, P. M., "Emerging enhanced imaging technologies of the esophagus: Spectroscopy, confocal laser endomicroscopy, and optical coherence tomography," Journal of Surgical Research 195(2), 502-514 (2015).

[6] Thosani, N., Abu Dayyeh, B. K., Sharma, P., Aslanian, H. R., Enestvedt, B. K., Komanduri, S., Manfredi, M., Navaneethan, U., Maple, J. T., Pannala, R., Parsi, M. A., Smith, Z. L., Sullivan, S. A., and Banerjee, S., "ASGE Technology Committee systematic review and meta-analysis assessing the ASGE Preservation and Incorporation of Valuable Endoscopic Innovations thresholds for adopting real-time imaging-assisted endoscopic targeted biopsy during endoscopic surveillance of Barrett's esophagus," Gastrointestinal Endoscopy 83(4), 684-698 (2016).

[7] Waterhouse, D. J., Fitzpatrick, C. R. M., di Pietro, M., and Bohndiek, S. E., "Emerging Optical Methods for Endoscopic Barrett's Surveillance," The Lancet Gastroenterology 83 Hepatology, In Press (2018).

[8] Perelman, L. T., Backman, V., Wallace, M., Zonios, G., Manoharan, R., Nusrat, A., Shields, S., Seiler, M., Lima, C., Hamano, T., and others, "Observation of periodic fine structure in reflectance from biological tissue: A new technique for measuring nuclear size distribution," Physical Review Letters 80(3), 627 (1998).

[9] Gora, M. J., Suter, M. J., Tearney, G. J., and Li, X., "Endoscopic optical coherence tomography: Technologies and clinical applications [Invited]," Biomedical Optics Express 8(5), 2405 (2017).

[10] Perelman, L. T. and Backman, V., "Light Scattering Spectroscopy of Epithelial Tissues: Principles and Applications," in [Handbook of Optical Biomedical Diagnostics, Second Edition, Volume 2: Methods], SPIE PRESS (2016).

[11] Pyhtila, J. W., Chalut, K. J., Boyer, J. D., Keener, J., D’Amico, T., Gottfried, M., Gress, F., and Wax, A., "In situ detection of nuclear atypia in Barrett's esophagus by using angle-resolved low-coherence interferometry," Gastrointestinal Endoscopy 65(3), 487-491 (2007).

[12] Ughi, G. J., Gora, M. J., Swager, A.-F., Soomro, A., Grant, C., Tiernan, A., Rosenberg, M., Sauk, J. S., Nishioka, N. S., and Tearney, G. J., "Automated segmentation and characterization of esophageal wall in vivo by tethered capsule optical coherence tomography endomicroscopy," Biomedical Optics Express 7(2), 409 (2016).

[13] Qiu, L., Pleskow, D. K., Chuttani, R., Vitkin, E., Leyden, J., Ozden, N., Itani, S., Guo, L., Sacks, A., Goldsmith, J. D., Modell, M. D., Hanlon, E. B., Itzkan, I., and Perelman, L. T., "Multispectral scanning during endoscopy guides biopsy of dysplasia in Barrett's esophagus," Nature Medicine 16(5), 603-606 (2010).

[14] Wang, Z., Tangella, K., Balla, A., and Popescu, G., "Tissue refractive index as marker of disease," Journal of biomedical optics 16(11), 116017-1160177 (2011).

[15] Gordon, G. S. D., Joseph, J., Bohndiek, S. E., and Wilkinson, T. D., "Single-Pixel Phase-Corrected Fiber Bundle Endomicroscopy With Lensless Focussing Capability," J. Lightwave Technol. 33(16), 3419-3425 (2015).

[16] Sibille, A. and Lenz, P., "Ex Vivo Clinical Measurement of the Optical Penetration Depth in Digestive Tissues," Lasers in medical science 13(4), 283-287 (1998).

[17] Ackroyd, R., Brown, N. J., Stephenson, T. J., Stoddard, C. J., and Reed, M. W., "Ablation treatment for Barrett oesophagus: What depth of tissue destruction is needed?," Journal of Clinical Pathology 52(7), 509-512 (1999).

[18] Goodman, J. W., [Introduction to Fourier Optics], W.H. Freeman, Macmillan Learning, New York, fourth edition ed. (2017). 
[19] Voelz, D. G., [Computational Fourier Optics: A MATLABß Tutorial], SPIE, 1000 20th Street, Bellingham, WA 98227-0010 USA (2011).

[20] Gerchberg, R. and Saxton, W., "A practical algorithm for the determination of phase from image and diffraction plane pictures," Optik 35(2), 237-246 (1972).

[21] Fienup, J. R., "Phase retrieval algorithms: A comparison," Applied Optics 21(15), 2758 (1982).

[22] Wang, L., Dong, B., and Yang, G., "Phase retrieval from two intensity measurements in an optical system involving nonunitary transformation," Applied Optics 29(23), 3422 (1990). 